\title{
The role of 18-FDG PET/CT assessment of functional brain metabolism in cancer patients after chemotherapy
}

\author{
Shady Mohamed Tarek Gamal ${ }^{1 *}$, Amr Osama M. A. Azab ${ }^{1}$, Sherif Mohamed El Refaei ${ }^{2}$ and Mohamed Houseni ${ }^{3}$
}

\begin{abstract}
Background: Most neuropsychological studies on chemotherapy (CHT)-treated cancer survivors reported cognitive impairments in multiple domains such as executive functions, learning, memory, attention, verbal fluency, and speed of information processing. The CHT effects range from small to moderate, involving mostly the cognitive functions sub-served by frontal lobe. This study aimed to evaluate the role of PET/CT in the assessment of the effect of chemotherapy on the glucose metabolism in the brain in cancer patients after the chemotherapy treatment.
\end{abstract}

Results: This was a prospective study carried out in 2 years for patients who have done PET/CT scans for assessment of the change of the glucose uptake in the brain in pre- and in post-therapeutic state. A total number of 30 patients, 8 males and 22 females, were examined. The age of the patients ranged from 29 to 79 years (mean 57.9). Each patient underwent at least two PET/CT scans, first before the initiation of the therapy, and second was at least 3 months after starting the chemotherapy regimen. This study employed an adaptive threshold method, SCEN IUM version 2.0.1. Automatic ROI identification was performed through around 10 regions of the brain. After segmentation of FDG uptake in the different brain regions of each subject, we measured average glucose uptake (SUVmean), registered by SCENIUM software.

Conclusion: There was significant reduction in the brain metabolism "FDG uptake" in all regions of the brain, mainly at the mesial temporal lobes as well as the frontal lobes. This metabolic change proves that chemotherapy has an adverse effect on the brain that can be objectively assessed with modern imaging techniques.

Keywords: PET/CT, Brain, Metabolism, Chemotherapy, Cognitive

\section{Background}

Adjuvant chemotherapy (CHT) in cancer patients can cause cognitive changes; studies have started at around mid-1970s with systematic research starting in the early 1990s [1]. Most of the studies on the CHTtreated cancer patients reported impairments regarding the cognitive functions in multiple aspects such as learning, attention, memory, and verbal abilities. The CHT effects have a wide range with reported studies stating a range from small to moderate effects;

\footnotetext{
* Correspondence: shady_trister@hotmail.com

${ }^{1}$ Radiodiagnosis, Faculty of Medicine, Cairo University, Giza, Egypt

Full list of author information is available at the end of the article
}

the cognitive functions are seen mostly involving the frontal lobes [2]. A diminution in certain brain functions have been reported in some patients after adjuvant cancer chemotherapy or even during the therapy course. Such phenomenon has been observed in CHT-treated patients for brain cancer as well as in patients with cancers in peripheral locations, such as the breast [3]. The diminution of the cognitive functions affected about one third of those patients. It is now commonly known as "chemo-fog" [4]. The chemo-fog phenomenon has been reported as observed cognitive changes occurring in a group of patients who are treated with chemotherapy to inhibit 
and stop the fatal occult metastatic diseases which may appear even after years of stopping the therapy. The domains regarding the cognitive impairments include language, multitasking, and information organization. Many studies reported occurrence of the chemo-fog phenomena in patients who received chemotherapy for cancer treatment [5]. A study stated cognitive impairment as a common feature in 50 consecutive cancer patients in their hospital. Another found cognitive function impairments in patients with breast cancer at its severest form 2 years after ending the therapy in a sample of patients who have been treated with high-dose of chemotherapy [6].

The study aimed to evaluate the role of PET/CT in the assessment of the effect of chemotherapy on the glucose metabolism in the brain in cancer patients after the chemotherapy treatment.

\section{Methods}

This was a prospective study carried out over the span of 2 years for patients who underwent PET/CT scans for assessment of the change of the glucose uptake in the brain in pre- and in post-therapeutic state.

A total number of 30 patients, 8 males and 22 females, were examined. The age of the patients ranged from 29 to 79 years, and the mean was 57.9. All patients were diagnosed with primary tumors as discussed in (Table 1) with none of them had primary or secondary brain lesions.

All the patients underwent two PET/CT scans, the first before commencing the chemotherapy, and the second after ending the chemotherapy regimen.

\section{Patients' selection}

The selection process included patients who fulfilled the criteria given below and approved by the ethical committee.

\section{Inclusion criteria}

- Patients of any age group diagnosed with a primary tumor whose treatment plan entails systemic chemotherapy.

\section{Exclusion criteria}

- Blood glucose level $>200 \mathrm{mg} / \mathrm{ml}$ at the time of examination that fails to be controlled.

- Patients received radiation therapy over the head and neck region.

\section{Medical data}

$\triangleright$ Personal history: gender, age, smoking, height, weight, and body mass index. History of the primary
Table $1 \mathrm{CT}$ findings in the 30 patients of study population

\begin{tabular}{|c|c|c|c|}
\hline \multirow[t]{11}{*}{ Primary } & Adenocarcinoma of the spine & 1 & $3.3 \%$ \\
\hline & Ovarian cancer & 1 & $3.3 \%$ \\
\hline & Breast cancer & 9 & $30.0 \%$ \\
\hline & Bronchogenic carcinoma & 8 & $26.6 \%$ \\
\hline & Colonic cancer & 1 & $3.3 \%$ \\
\hline & Hepatic focal lesions & 1 & $3.3 \%$ \\
\hline & Lacrimal gland neoplasm & 1 & $3.3 \%$ \\
\hline & Lymphoma & 4 & $13.3 \%$ \\
\hline & Palatal melanoma & 1 & $3.3 \%$ \\
\hline & Parotid neoplasm & 2 & $6.7 \%$ \\
\hline & $\mathrm{RCC}$ & 1 & $3.3 \%$ \\
\hline
\end{tabular}

neoplasm, the duration of the chemotherapeutic agent, and any associated systemic disease like diabetes mellitus or hypertension. Any patient receiving adjuvant radiotherapy or hormonal treatment or who underwent surgical removal of the primary neoplasm

$\triangleright$ Present illness

Primary indication for PET

Stage of primary neoplasm

$\triangleright$ The date of the previous investigation and the general neurological state of the patient

$\triangleright$ General examination: Pulse rate, blood pressure, and random blood sugar

\section{Image protocol}

All exams performed using one of the following systems Philips Gemini TF (Time-of-Flight) PET/CT machine equipped with LYSO crystals (Philips, Holland) and 64 slices CT, Philips Gemini GXL 16 PET/CT, and Siemens Biograph mCT20 Excel PET/CT.

\section{Patient preparation}

Patients were instructed to fast for at least $6 \mathrm{~h}$ and were asked to avoid strenuous exercise for $24 \mathrm{~h}$ prior to the examination. Blood glucose was checked in all patients prior to FDG injection. Serum blood glucose levels were determined by finger stick measurement before administration of the radiotracer to ensure a serum glucose level below $200 \mathrm{mg} / \mathrm{dl}$. All patients were kept in a warm and calm environment, then received $\sim 5.2 \mathrm{MBq}$ of ${ }^{18} \mathrm{~F}$-FDG per kilogram of body weight intravenously.

\section{Image technique}

Each patient underwent multiple time-point ${ }^{18}$ F-FDG PET CT imaging at $~ 60$ and $180 \mathrm{~min}$ after the administration of ${ }^{18}$ F-FDG. At $60 \mathrm{~min}$, the acquisition time per bed position was $2 \mathrm{~min}$. To compensate for radioactive decay of the tracer, the acquisition time per bed position was extended to $4 \mathrm{~min}$ for the 3 -h scan. 
Table 2 The mean, standard deviation (SD), median, minimum, and maximum of the different regions before (Pre) and after (Post) chemotherapy

\begin{tabular}{|c|c|c|c|c|c|c|c|c|c|c|c|}
\hline & \multicolumn{5}{|l|}{ Pre } & \multicolumn{5}{|l|}{ Post } & \multirow[t]{2}{*}{$P$ value } \\
\hline & Mean & SD & Median & Minimum & Maximum & Mean & SD & Median & Minimum & Maximum & \\
\hline BG (R) & 11.91 & 11.17 & 7.96 & 2.59 & 48.90 & 7.87 & 5.09 & 8.14 & 0.57 & 20.02 & 0.03 \\
\hline BG (L) & 11.79 & 11.11 & 7.79 & 2.84 & 48.20 & 7.74 & 5.49 & 7.08 & 0.71 & 22.70 & 0.015 \\
\hline Cerbellum (R) & 9.98 & 9.60 & 6.45 & 2.40 & 37.40 & 5.99 & 3.72 & 6.95 & 0.26 & 12.22 & 0.014 \\
\hline Cerbellum (L) & 10.56 & 9.54 & 6.95 & 2.88 & 36.00 & 7.21 & 6.27 & 7.08 & 0.63 & 33.46 & 0.005 \\
\hline CF (R) & 13.41 & 12.44 & 9.19 & 2.68 & 45.60 & 8.77 & 5.71 & 8.41 & 0.69 & 20.83 & 0.028 \\
\hline CF (L) & 13.80 & 13.04 & 9.30 & 2.02 & 50.50 & 8.97 & 6.98 & 7.64 & 0.66 & 32.80 & 0.009 \\
\hline $\mathrm{FL}(\mathrm{R})$ & 11.35 & 10.29 & 7.24 & 2.20 & 39.30 & 6.68 & 4.30 & 6.09 & 0.73 & 16.66 & 0.002 \\
\hline $\mathrm{FL}(\mathrm{L})$ & 11.71 & 10.94 & 7.46 & 2.70 & 45.10 & 7.18 & 5.15 & 6.11 & 1.10 & 22.69 & 0.003 \\
\hline $\mathrm{TL}(\mathrm{R})$ & 10.55 & 9.25 & 7.49 & 1.64 & 37.52 & 6.70 & 4.23 & 7.30 & 0.25 & 15.85 & 0.004 \\
\hline $\mathrm{TL}(\mathrm{L})$ & 10.27 & 8.84 & 6.97 & 2.52 & 35.71 & 7.91 & 6.90 & 7.34 & 1.10 & 37.67 & 0.041 \\
\hline$C R(R)$ & 13.58 & 16.21 & 7.04 & 1.82 & 77.87 & 7.89 & 9.60 & 4.03 & 0.63 & 51.78 & $<0.001$ \\
\hline CR (L) & 14.02 & 16.36 & 7.29 & 3.20 & 72.80 & 8.36 & 10.53 & 5.10 & 0.90 & 52.23 & $<0.001$ \\
\hline CG (R) & 13.46 & 16.12 & 7.30 & 2.40 & 78.20 & 7.78 & 8.61 & 6.30 & 1.00 & 48.90 & 0.004 \\
\hline CG (L) & 13.98 & 17.11 & 7.40 & 2.50 & 79.40 & 7.92 & 9.22 & 5.95 & 0.70 & 51.73 & 0.001 \\
\hline MT (R) & 10.84 & 11.96 & 5.53 & 1.64 & 56.12 & 7.68 & 8.36 & 6.36 & 0.60 & 36.56 & 0.037 \\
\hline MT (L) & 11.00 & 12.22 & 5.64 & 1.61 & 55.65 & 7.50 & 8.29 & 6.46 & 0.60 & 36.87 & 0.026 \\
\hline $\mathrm{OL}(\mathrm{R})$ & 14.99 & 16.76 & 7.94 & 1.00 & 80.50 & 9.23 & 8.98 & 8.90 & 0.50 & 49.50 & 0.007 \\
\hline $\mathrm{OL}(\mathrm{L})$ & 14.94 & 16.82 & 7.84 & 1.87 & 81.00 & 9.88 & 10.35 & 8.35 & 0.90 & 51.30 & 0.007 \\
\hline PL (R) & 12.38 & 15.61 & 7.50 & 1.00 & 78.10 & 7.69 & 8.60 & 6.35 & 0.60 & 47.90 & 0.006 \\
\hline PL (L) & 12.37 & 15.73 & 7.31 & 1.10 & 79.60 & 8.35 & 10.45 & 5.63 & 0.80 & 50.51 & 0.005 \\
\hline
\end{tabular}

$B G$ basal ganglia, $C R$ central region, $C G$ cingulate gyrus, $M T$ mesial temporal lobe, $O L$ occipital lobe, $F L$ frontal lobe, $T L$ temporal lobe, $C F$ calcarine fissure, $P L$ parietal lobe

Low-dose non-enhanced CT scan first was done first, then a whole brain PET study followed by diagnostic enhanced CT scan. The whole study took approximately $15 \mathrm{~min}$. The contrast enhanced helical CT was performed following injection of $1-2 \mathrm{ml} / \mathrm{kg}$ of a lowosmolarity iodinated contrast medium at a rate of $4 \mathrm{ml} / \mathrm{s}$ by using a power injector. For a typical brain PET/CT study, scanning began at the level of the skull vertex to base. The total length of CT coverage was an integral number of bed positions scanned during acquisition of PET data. The study was performed with the patient breathing quietly. Typical scanning parameters would be a collimator width of $5.0 \mathrm{~mm}$, pitch of 1.5 , gantry rotation time of $0.8 \mathrm{~s}$, and field of view of $50 \mathrm{~cm}$. The helical data are retrospectively reconstructed at $1-\mathrm{mm}$ intervals.

PET was performed following the CT study without moving the patient. Approximately six to seven bed positions are planned in the three-dimensional acquisition mode for scanning the entire patient with 3-5min acquisition at each bed position.

Brain PET was acquired at a single bed position planned in the three-dimensional acquisition mode for scanning the whole brain at 5-min acquisition; at this bed position, attenuation correction images were taken to determine the exact structural location of FDG uptake in the brain. Many trans-axial PET and CT images were first reconstructed. These are then reformatted into coronal and sagittal images to facilitate image interpretation. For each of these sets of PET and CT images, corresponding "fusion" images, combining the two types of data, also were generated.

The whole acquisition time for an integrated PET/ CT scan was approximately $25 \mathrm{~min}$. PET image data sets were reconstructed using CT data for attenuation correction and co-registered images were displayed using special software.

\section{Image analysis}

Images were acquired using a low-dose technique for attenuation correction of PET images, and to define cortical metabolic activity, an adaptive threshold method, SCEN IUM version 2.0.1, was used. Automatic ROI identification was performed through around 10 regions of the brain including the cerebral cortex, as well as basal ganglia of both cerebral hemispheres and cerebellum separately (right and left). Partial volume correction was implemented for each 
Table 3 The correlation between percentage change of the left frontal lobe with the sex of the patients

\begin{tabular}{|c|c|c|c|c|c|c|c|c|c|c|c|}
\hline & \multicolumn{10}{|l|}{ Sex } & \multirow{3}{*}{$\begin{array}{l}P \\
\text { value }\end{array}$} \\
\hline & \multicolumn{5}{|l|}{ Male } & \multicolumn{5}{|l|}{ Female } & \\
\hline & Mean & $\begin{array}{l}\text { Standard } \\
\text { Deviation }\end{array}$ & Median & Minimum & Maximum & Mean & $\begin{array}{l}\text { Standard } \\
\text { Deviation }\end{array}$ & Median & Minimum & Maximum & \\
\hline FL (L) \% change & 6.71 & 53.45 & -3.17 & -76.15 & 108.59 & -28.15 & 51.38 & -39.79 & -86.42 & 132.40 & 0.040 \\
\hline
\end{tabular}

measurement to account for underestimation of SUV values. After segmentation of FDG uptake in the different brain regions of each subject, we measured average glucose uptake (SUVmean), registered by SCENIUM software.

The pre and post therapy studies of every patient were conducted on the same PET scanner, all the studies were analyzed by the same software, the software automatically recorded the readings of the different regions as previously described, and the different readings were recorded twice on both sides of the brain.

A consultant radiologist and consultant nuclear medicine physician each with about 20 years' experience interpreted all images independently. First, each study was reviewed separately; then, the fused images were evaluated.

Table 4 The correlation between percentage change of the above-examined regions and the time interval in weeks

\begin{tabular}{ll}
\hline Region percentage change (\%) & $P$ value \\
\hline BG (R) & 0.013 \\
BG (L) & 0.015 \\
Cerebellum (R) & 0.001 \\
Cerebellum (L) & 0.017 \\
CF (R) & 0.316 \\
CF (L) & 0.391 \\
CR (R) & 0.042 \\
CR (L) & 0.022 \\
CG (R) & 0.007 \\
CG (L) & 0.004 \\
FL (R) & 0.013 \\
FL (L) & 0.109 \\
TL (R) & 0.059 \\
TL (L) & 0.293 \\
OC (R) & 0.013 \\
OC (L) & 0.056 \\
PL (R) & 0.014 \\
PL (L) & 0.021 \\
MT (R) & 0.038 \\
MT (L) & 0.079 \\
\hline BG basa & \\
\hline &
\end{tabular}

$B G$ basal ganglia, $C R$ central region, $C G$ cingulate gyrus, $M T$ mesial temporal lobe, $O L$ occipital lobe, $F L$ frontal lobe, $T L$ temporal lobe, $C F$ calcarine fissure, $P L$ parietal lobe
The total reading of all regions of the brain for each patient was summarized into two groups (before and after ending the chemotherapy treatment). The percentage changes in each patient was calculated and correlated with other factors including the sex, systemic disease, radiotherapy, hormonal therapy, and the time interval between the two sessions of the chemotherapy (Tables 2, 3, and 4).

\section{Statistical methods}

The mean, standard deviation, minimum and maximum of the total 10 different regions readings of the 30 patients were recorded in the pre- and posttherapeutic state before a consensus report was approved by both consultants.

The percentage change in each region was also calculated and compared in the pre- and postchemotherapeutic studies, and the percentage change in both pre- and post-therapeutic studies were also correlated with other factors in each patient including the gender, the other therapies (hormonal or radiotherapy), and systemic diseases such as diabetes mellitus and the systemic hypertension.

The time interval between the chemotherapy cycles as well as the last chemotherapy cycle was also correlated with the percentage changes.

Data were coded and entered using the statistical package SPSS (Statistical Package for the Social Sciences) version 25. Data were summarized using mean, standard deviation, median, minimum, and maximum in quantitative data and using frequency (count) and relative frequency (percentage) for categorical data. Comparisons between quantitative variables were done using the non-parametric Mann-Whitney test. For comparison of serial measurements within each patient, the nonparametric Wilcoxon signed-rank test was used.

Correlations between quantitative variables were done using the Spearman correlation coefficient. $P$ values less than 0.05 were considered as statistically significant.

\section{Results}

Comparison was made between the same brain regions before and after ending the chemotherapy treatment for each patient, and the $P$ values were calculated as demonstrated in Table 2. 
These regions included the basal ganglia, cerebellum, central regions, Cingulate gyri, mesial temporal lobes, occipital lobes, frontal lobes, temporal lobes, calcarine fissure, and parietal lobes.

In this study, there was significant decrease in glucose metabolism in the all 10 different ROIs before and after the chemotherapy as signified by the $P$ values $<0.05$ (Figs. 1, 2, and 3).

The central and cingulate regions as well as the frontal and temporal lobes were among the most affected areas by the chemotherapy treatment; other regions were significantly affected as well but showed less affection compared to the forementioned four groups.

No significant correlation of percentage change among females and males apart from mild significant reduction in glucose uptake change in the left frontal lobe region among females (Table 3).

There was no significant correlation regarding the percentage change for the patients who had systemic disease or received radio or hormonal therapy.

There was significant direct relationship between the percentage change and the time interval between the last cycle and the second examination. Those changes are seen at both ganglia regions, the cerebellar hemispheres,

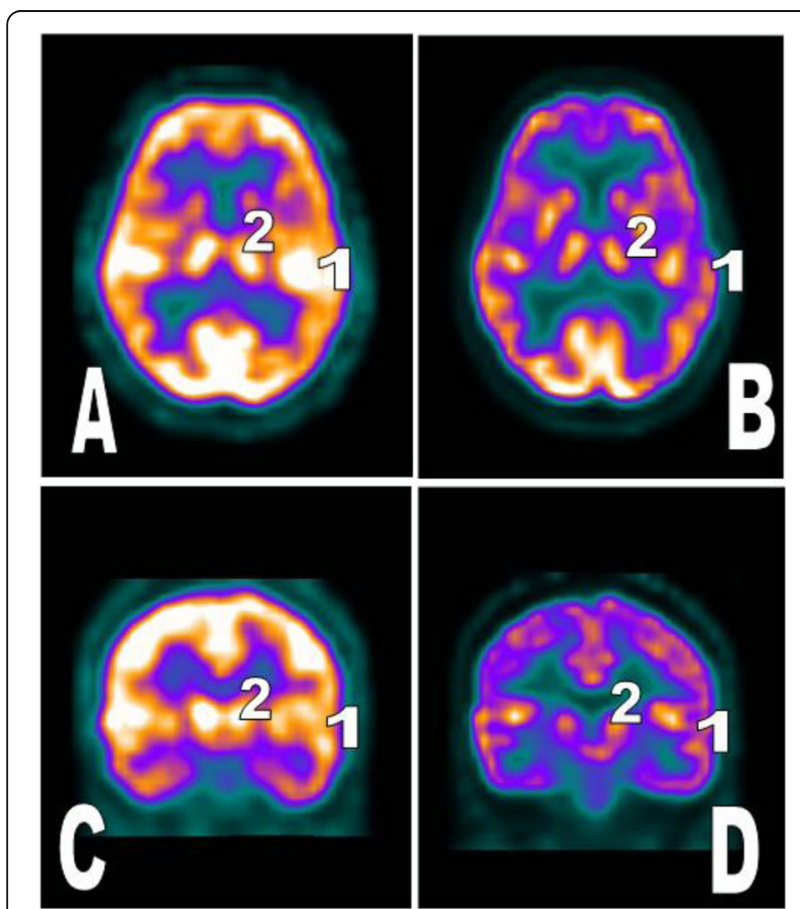

Fig. 1 Seventy-one-year-old female discovered to have colon cancer by prior $\mathrm{CT}$, the patient came for PET/CT assessment. Fused coronal and axial PET/CT images show reduced FDG uptake in the post chemotherapeutic state (images $\mathbf{b}$ and $\mathbf{d}$ ) compared to the prechemotherapeutic state (images $\mathbf{a}$ and $\mathbf{c}$ ) along the left temporal lobes $\{1\}$ (SUVmean 29 compared to 8.5) and the left basal ganglia \{2\} (SUVmean 25 compared to 6.5)

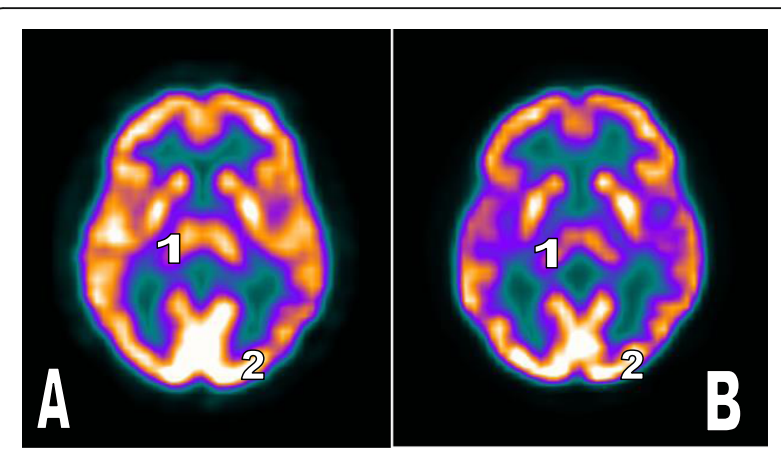

Fig. 2 Sixty-one-year-old female discovered to have breast carcinoma by prior $\mathrm{CT}$, the patient came for PET/CT assessment. $\mathbf{a}, \mathbf{b}$ Axial fused PET/CT images of the brain before and after

chemotherapy. Fused axial PET/CT images show reduced FDG uptake in the post chemotherapeutic state (image B) compared to the pre-chemotherapeutic state (image $\mathbf{a}$ ) along the right thalamus $\{1\}$ (SUVmean 10.4 compared to 11.5) and the left occipital lobe $\{2\}$ (SUVmean 9.8 compared to 13.3)

the central regions, the right frontal lobe, the right mesial temporal lobe, the right occipital lobe, and both parietal lobes (Table 4).

\section{Discussion}

This study included 30 patients aiming at evaluation of the effect of chemotherapy on the brain metabolism after the chemotherapy.

The study concluded that that there was significant reduction of the glucose uptake in all the 10 ROIs after chemotherapy. Those changes were mostly evident at the central and cingulate regions, this was comparable to the study of Horky et al. [7]. The cingulate gyrus being the part of the brain mainly controlling the memory, behavioral, and the emotional brain functions [8], so the metabolic effect of the chemotherapeutic agents on this

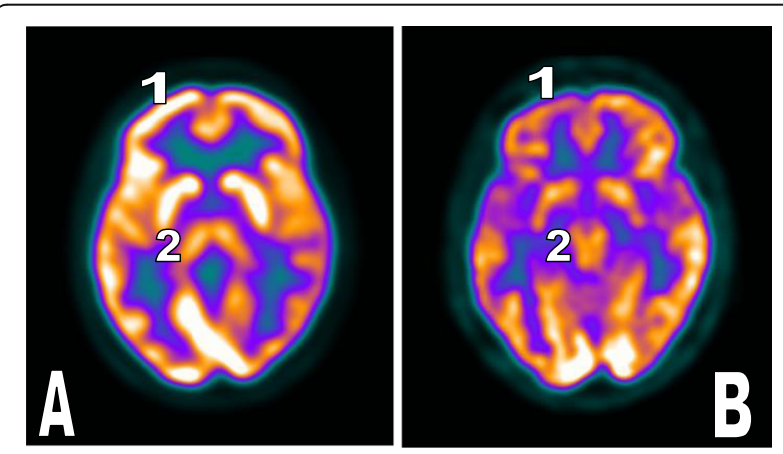

Fig. 3 Fifty-nine-year-old female discovered to have lacrimal gland neoplasm by prior $C T$, the patient came for PET/CT assessment. Axial fused PET/CT images of the brain before and after chemotherapy. Fused axial PET/CT images show reduced FDG uptake in the post chemotherapeutic state (image b) compared to the prechemotherapeutic state (image a) along the right frontal lobes $\{1\}$ (SUVmean 11.3 compared to 20) and along the right basal ganglia regions $\{2\}$ (SUVmean 12.4 compared to 23.2) 
part of the brain specifically could explain the forementioned functional deteriorations following treatment.

The second most affected regions were the frontal lobes with $P$ values $<0.002$; the frontal lobes play an important part in integrating longer non-task-based memories stored across the brain [9], another evidence that support the effect on the chemotherapeutic agents upon the memory function of the brain.

The third most affected regions were the temporal lobes (specially the right) with $P$ values $<0.004$. The temporal lobe communicates with the hippocampus and plays a key role in the formation of explicit long-term memory modulated by the amygdala [10].

All the other regions showed also significant reductions in the brain metabolism but with less evident $P$ values. These regions include the basal ganglia, the cerebellar hemispheres, the right frontal lobe, the right mesial temporal lobe, the right occipital lobe, and both parietal lobes. Those regions are responsible for memory, visual, and behavioral changes.

There was also a significant correlation between the time intervals between the last chemotherapy session and the second examination in certain regions suggesting the long-term effect of the chemotherapy on the brain functions as stated by Pendergrass et al. [11] that may continue to affect the brain functions for long time. In correlation with other factors including the sex as well as the systemic diseases, there was no significant difference which is comparable to the results of Sorokin et al. [12]. This could be attributed to the small sample size.

The results of our study proves that the chemotherapy has an adverse effect on the brain that can be objectively and early detected by FDG PET examination as a functional molecular imaging tool based on quantitative measurement of the functional alterations before any morphological changes could be identified. However, the results are still preliminary and we admit that the limited sample of our study, the lack of specificity regarding the primary neoplasm, the chemotherapeutic regimen, the non-constant number of treatment courses, and interval between the chemotherapy course and post treatment scan as well as the lack of repeated follow-up over longer periods and coupling with detailed clinical assessment and profile of the patients before and after treatment are the main limitations of our study and the few preceding clinical trials.

At this point, our work may open the window for further studies with larger population and targeted specific chemotherapeutic agents and particular age groups with consideration to pre-existing medical diseases and assessment of their clinical profile before and after chemotherapy in order to predict and minimize or delay such therapy-related cognitive impairment by selecting the most appropriate protocols and implementing supportive measures so as to ensure better quality of life of those patients. Moreover combined and comparative assessment of those factors by both FDG PET and resting state functional MRI may give an insight on the correlation between the metabolic and neural network alterations in those patients.

\section{Limitations of the study}

The exact type of the chemotherapy and the affected cognitive domains were lacking in this study.

\section{Conclusion}

Chemotherapy can cause cognitive and psychosocial complications; functional imaging could play a pivotal role.

$\mathrm{PET} / \mathrm{CT}$ has the potential to serve as an early marker for cognitive changes associated with chemotherapy.

This study found a significant reduction in the brain metabolism "FDG uptake" in all regions of the brain, mainly at the mesial temporal lobes as well as the frontal lobes. This metabolic change proves that chemotherapy has an adverse effect on the brain that can be objectively assessed with modern imaging techniques.

\section{Abbreviations}

CT: Computed tomography; FDG: Fluorodeoxyglucose; PET: Positron emission tomography; ROIs: Regions of interest; SD: Standard deviation; SUV: Standard uptake value; SUVmax: Maximum SUV; SUVmin: Minimum SUV;

SUVmean: Mean SUV; CHT: Chemotherapy; RCC: Renal cell carcinoma

\section{Acknowledgements}

Not applicable.

\section{Authors' contributions}

S.M.T put the idea of the study. Editor of the manuscript. Participated in the study design. A.O.A, S.R., and M.H. participated in the study design, patients' collection, and clinical assessment and performed the statistical analysis. All authors read and approved the final manuscript.

\section{Funding}

Not applicable (no funding received for this study).

\section{Availability of data and materials}

All the datasets used and analyzed in this study are available with the corresponding author on reasonable request.

\section{Ethics approval and consent to participate}

Written informed consent was signed by all patients before the examination. The study was approved by the research committee of Faculty of Medicine, Kasr Alainy Hospital, Cairo University 2020. No reference number was provided as the committee just say yes or no according to the system in our Faculty of Medicine at 2017 (date of starting of this research).

\section{Consent for publication}

All patients included in this research were fully conscious and older than 16 years old and gave written informed consent to publish the data contained within this study. 


\section{Competing interests}

The authors declare that they have no competing interests.

\section{Author details}

${ }^{1}$ Radiodiagnosis, Faculty of Medicine, Cairo University, Giza, Egypt. ${ }^{2}$ Nuclear Medicine, Faculty of Medicine, Cairo University, Giza, Egypt. ${ }^{3}$ Radiodiagnosis, National Liver Institute, Menoufia University, Shibin El Kom, Egypt.

Received: 2 October 2020 Accepted: 27 December 2020

Published online: 08 January 2021

\section{References}

1. Baudino B, D'agata F, Caroppo P et al (2012) The chemotherapy long-term effect on cognitive functions and brain metabolism in lymphoma patients. Q J Nucl Med Mol Imaging 56(6):559-568

2. Falleti MG, Sanfilippo A, Maruff $P$ et al (2005) The nature and severity of cognitive impairment associated with adjuvant chemotherapy in women with breast cancer: a meta-analysis of the current literature. Brain Cogn 59: 60-70

3. Olin JJ (2001) Cognitive function after systemic therapy for breast cancer. Oncology (Williston Park) 15(5):613-618 discussion 618, 621-4

4. Tannock IF, Tim AA, Ganz PA et al (2004) Cognitive impairment associated with chemotherapy for cancer: report of a workshop. J Clin Oncol 22: 2233-2239

5. Meyers CA (2000) Neurocognitive dysfunction in cancer patients. Oncology (Williston Park) 14(1):75-79 discussion 79, 81-2, 85

6. DeVita VT Jr et al (2015) Principles \& practice of oncology, 10th edn. Wolters Kluwer Health Lippincott Williams \& Wilkins, Philadelphia

7. Horky LL, Gerbaudo VH, Zaitsev A et al (2014) Systemic chemotherapy decreases brain glucose metabolism. Div Nucl Med Dep Radiol 1(10): 788-798

8. Kozlovskiy S, Vartanov A, Pyasik M et al (2013) Anatomical characteristics of cingulate cortex and neuropsychological memory tests performance. Procedia Soc Behav Sci 08:537

9. Kosslyn S (2007) Cognitive psychology: mind and brain. Prentice Hall, New Jersey pp. 21, 194-199, 349

10. Kimberg DY, Farah MJ (1993) A unified account of cognitive impairments following frontal lobe damage: the role of working memory in complex, organized behavior. J Exp Psychol Gen 122(4):411-428. https://doi.org/10. 1037//0096-3445.122.4.411 PMID 8263463

11. Pendergrass CJ, Targum SD, Harrison JE (2018) A cognitive impairment associated with cancer. Innov Clin Neurosci 15(1-2):1PMC5819720

12. Sorokin J, Saboury B, Ahn JHA et al (2014) Adverse functional effects of chemotherapy on whole-brain metabolism: a PET/CT quantitative analysis of FDG metabolic pattern of the "chemo-brain". Clin Nucl Med 39(1):e35-e39. https://doi.org/10.1097/RLU.0b013e318292aa81

\section{Publisher's Note}

Springer Nature remains neutral with regard to jurisdictional claims in published maps and institutional affiliations.

\section{Submit your manuscript to a SpringerOpen ${ }^{\circ}$ journal and benefit from:}

- Convenient online submission

- Rigorous peer review

- Open access: articles freely available online

- High visibility within the field

- Retaining the copyright to your article

Submit your next manuscript at $\boldsymbol{\nabla}$ springeropen.com 